\title{
THE DEPENDENCE OF RADAR TARGET DETECTABILITY ON ARRAY WEIGHTING FUNCTION
}

\author{
C.M. Alabaster*, E.J. Hughes* \\ *Cranfield University, U.K. Email c.m.alabaster@cranfield.ac.uk
}

Keywords: radar detection, clutter, medium PRF, illumination function.

\section{Draft Paper}

This paper describes simulation work to assess the detectability of targets by an airborne fire control radar (FCR) operating in a medium pulse repetition frequency (PRF) mode in the presence of strong ground clutter as a function of transmitting and receiving array weighting functions. It describes the radar, antenna and clutter modelling for a system operating a 3 of 8 medium PRF schedule waveform. Medium PRF waveforms and the selection of PRFs are described in the authors' previous papers [1,2,3,4,6].

Target detectability depends on the number of PRFs in which any target is visible and on the probability of detection (Pd) in each PRF. The Pd in each PRF is determined by the signal to noise plus clutter ratio (SNCR), amongst other factors, and varies across the range and Doppler detection space of the radar due to the ambiguous repetition of clutter across this detection space. Minimizing side lobe clutter (SLC) through the minimization of antenna sidelobe level is a design priority for such systems. This may be achieved by applying a tapered illumination function across the antenna aperture and can be implemented readily by appropriate amplitude and phase weightings of the elements of an electronically steerable phased array antenna. However, tapered illumination functions result in a reduction in main beam boresight gain together with a broadening of the main beam, both of which are further degraded when the beam is phase steered away from its mechanical boresight. Furthermore, phase steering tends to generate increased sidelobes. Thus there appears to be a conflict of interests in applying tapered illumination across an array antenna as far as target detection is concerned; on the one hand the tapered illumination reduces the sidelobe level but on the other it leads to a loss of main beam gain. Thus both clutter and target signal strengths are reduced through the use of a tapered antenna illumination or, conversely, both are maximized for a uniformly illuminated antenna. The question arises as to whether tapered illumination actually leads to increased target detectability or not.

This question has been addressed by modelling the clutter scene in an airborne FCR utilising 189 combinations of transmitting and receiving array weighting functions and operating conditions. The transmitting array functions considered were: uniform, radial transmit taper (RTT) and successive projection transmit nulling (SPTN) [5]. The receiving array illumination functions considered were: uniform, Taylor 35dB and Taylor 45dB. Seven combinations of transmitting and receiving array weighting functions (named patterns) were defined, as in Table 1.

\begin{tabular}{|c|c|c|}
\hline patterns & $\begin{array}{c}\text { Transmit } \\
\text { Weighting } \\
\text { Function }\end{array}$ & $\begin{array}{c}\text { Receive } \\
\text { Weighting } \\
\text { Function }\end{array}$ \\
\hline 1 & Uniform & Uniform \\
2 & Uniform & Taylor $35 \mathrm{~dB}$ \\
3 & Uniform & Taylor $45 \mathrm{~dB}$ \\
4 & RTT & Taylor $35 \mathrm{~dB}$ \\
5 & RTT & Taylor $45 \mathrm{~dB}$ \\
6 & SPTN & Taylor $35 \mathrm{~dB}$ \\
7 & SPTN & Taylor $45 \mathrm{~dB}$ \\
\hline
\end{tabular}

Table 1: Combinations of Array Weighting Functions

27 different operating conditions were also defined in terms of combinations of platform altitude $(1000 \mathrm{~m}$ and $5000 \mathrm{~m})$, azimuth scan angles $\left(0^{\circ}, 30^{\circ}\right.$ and $\left.60^{\circ}\right)$, elevation scan angle $\left(0^{0}, 6^{0}\right.$ down $)$ and probability of failed array elements $(0 \%$, $2 \%$ and $5 \%$ ). These conditions do not encompass the whole range of operating conditions but are a representative sample of them.

The detectability of targets in clutter was quantified using a "detectability map", [1] which provides a useful means of comparing target detectability as clutter conditions change. A detectability map denotes the minimum target RCS required for detection at each range and Doppler cell in an appropriate number of PRFs (i.e. three in this case). An example detectability map is illustrated in Figure 1. This illustrates a good schedule in which there are no blind velocities. There is, however, a thin strip of poor detectability along the bottom of the map and this corresponds to the region of the first eclipsed range. 


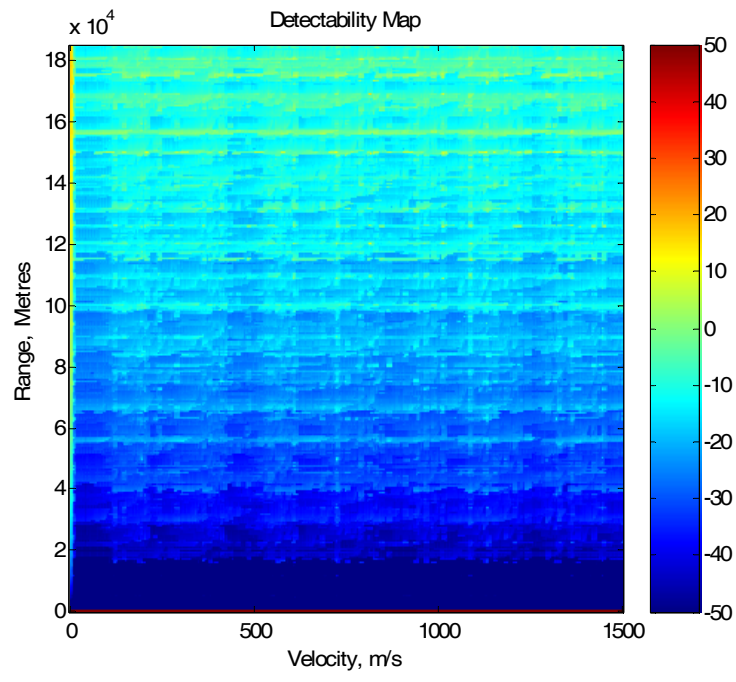

Figure 1: Detectability Map

Two metrics were devised to quantify target detectability from the detectability maps over the full range/Doppler detection space of the radar, namely, the ratio of comparisons $(X)$ and sum of differences comparison $(Y)$. The former is a measure of the relative area of the range/Doppler space for which the detectability of one test is greater than the detectability of another whereas the latter gives an impression of the "aggregate" level by which the detectability of one test is greater than the detectability of another. The combination of the two metrics, $X$ and $Y$, therefore indicates both the area extent of superiority of one detectability map over another and also on the aggregate margin of this superiority.

A comprehensive system of comparisons was derived to ensure a thorough test of all the various detectability maps. This ensured that the detectability maps of each of the seven combinations of transmitting and receiving weighting functions were compared with each of the others for all 27 combinations of operating conditions. These comparisons generated sets of statistics for the $X$ and $Y$ detectability metrics and the means of these statistics ( $\bar{X}$ and $\bar{Y}$ ) were generated across the 27 combinations of operating conditions. The means of the two metrics were used to provide a rank order of the seven combinations of transmitting and receiving array weighting functions from best target detectability to worst. Furthermore, a points scoring system was devised based on the rank order of the $\bar{X}$ and $\bar{Y}$ metrics. The results of the rank order points scoring is depicted in Figure 2. The "best" solution is the theoretical maximum number of points of 49 in each coordinate. From Figure 2 it is evident that patterns 5 and 7 are almost equal solutions which fall on a Pareto surface. However, of the two, patterns 7 offers the slightly better target detectability since its distance from the "best" is marginally less than that of patterns 5.

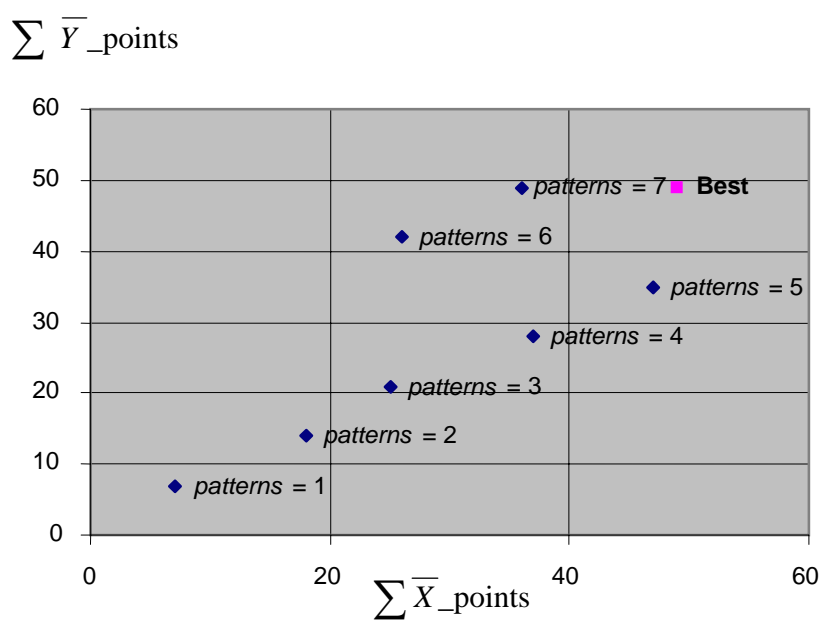

Figure 2: Points Positions of Solutions

The optimum combination of transmitting and receiving antenna array functions depend on which metric is used to quantify target detectability. Both metrics support the strategy of reducing the sidelobe levels on both transmitting and receiving antenna patterns through the use of tapered weighting functions. The ratio of comparisons $(X)$ metric of target detectability identifies that the best target detectability is to be obtained for the combination of the RTT on transmission and the Taylor $45 \mathrm{~dB}$ function on reception. $X$ seems to be most favourably influenced by the lower peak sidelobe levels of the RTT function on transmission, even to the extent that it identifies patterns $=4$ (RTT on transmit and Taylor 35dB on receive) as the second best combination and patterns $=7$ (SPTN on transmit and Taylor $45 \mathrm{~dB}$ on receive) as the third best combination. The sum of differences comparison $(Y)$ metric of target detectability identifies that the best target detectability is to be obtained for the combination of the SPTN function on transmission and the Taylor $45 \mathrm{~dB}$ on reception. $Y$ seems to be most favourably influenced by the lower average sidelobes of the SPTN, even to the extent that it identifies patterns $=6$ (SPTN on transmit and Taylor $35 \mathrm{~dB}$ on receive) as the second best combination and patterns $=5$ (RTT on transmit and Taylor $45 \mathrm{~dB}$ on receive) as the third best combination. However, both metrics agree that better target detectability is to be obtained by applying the Taylor $45 \mathrm{~dB}$ element weighting function to the receiving array over the corresponding Taylor $35 \mathrm{~dB}$ function.

Clearly, the differing metrics which one may use to quantify target detectability result in differing solutions with very little to chose between them. However, by combining the means of both the $X$ and $Y$ metrics in a points scoring system the best overall solution was identified as being the combination of the SPTN function on transmission and the Taylor $45 \mathrm{~dB}$ function on reception. This was very closely followed by the combination of the RTT function on transmission and the Taylor 45dB function on reception. The overall preference for the former may well be due to its lower average sidelobe levels on transmission. Nevertheless, it ought to be stressed that the margins between these two cases are very small and 
may very well be masked by statistical variations in noise, clutter and target RCS. It may also be worth noting that the RTT function results in an effective radiated power (ERP) some 0.6 dB higher than that of the SPTN function and so enjoys a small advantage in detection performance in noise limited cases. Furthermore, the RTT function (and its resulting beam pattern) is circularly symmetrical and so remains constant irrespective of the platform roll angle. The worst target detection performance was obtained when using the Uniform illumination function on the transmitting array. Indeed the test case of the Uniform function on both transmission and reception was found by both metrics to yield the worst target detection capability by a large margin.

\section{Acknowledgements}

This work was carried out as part of the UK MOD funded Output 3 Research Programme.

\section{References}

[1] C. M. Alabaster \& E. J. Hughes. "The Design of Medium PRF Radar Schedules For Optimum Detectability in Diverse Clutter Scenes” Proc. IEEE Waveform Diversity \& Design, Lihue, Kaua'i, HI, USA, $22^{\text {nd }}-27^{\text {th }}$ January 2006.

[2] C.M. Alabaster \& E.J. Hughes "Novel PRF Schedules for Medium PRF Radar”, Proc. Radar 2003, Adelaide, 3rd - 5th Sept. 2003.

[3] C.M. Alabaster, E.J. Hughes \& J.H. Matthew. "Medium PRF radar PRF Selection Using Evolutionary Algorithms”, IEEE Trans. Aerospace and Electronic Systems, vol. 39, no. 3, pp. 990-1001, July 2003.

[4] P. G. Davies and E. J. Hughes. "Medium PRF set selection using evolutionary algorithms", IEEE Transactions on Aerospace and Electronic Systems, vol. 38, no. 3, pp.933-939, July 2002.

[5] I. M. Mellor, F. J. Adams. "Benefits of Transmit Beam Pattern Synthesis for Airborne Phased Array Radar”, Proc. IRS 2005, Berlin, Germany, 6 - 8 Sept. 2005.

[6] D. A. Wiley, S. M. Parry, C.M. Alabaster and E. J. Hughes. "Performance Comparison of PRF Schedules for Medium PRF Radar", IEEE Trans. Aerospace and Electronic Systems. vol. 42, no. 2, Apr 2006, pp 601611. 